\title{
Dip Suspension Dosage Form
}

National Cancer Institute

\section{Source}

National Cancer Institute. Dip Suspension Dosage Form. NCI Thesaurus. Code C149436.

Liquid preparation consisting of a suspension intended for veterinary use by dipping. 